\title{
Clinical Effect of Knee Arthroscopy Combined with Liuwei Gubi Pill in Treating Middle-aged and Elderly Patients with Degenerative Meniscus Injury
}

\author{
Zike He, Kaifei He, Shangzeng Wang \\ Henan Provincial Hospital orthopaedics, Zhengzhou, Henan
}

\begin{abstract}
Objective: To study the effect of knee arthroscopy combined with Liuwei Gubi Pill on degenerative meniscus injury in middle-aged and elderly patients. Methods: A total of 92 middle-aged and elderly patients with degenerative meniscus injury who were treated in our hospital from August 2018 to February 2020 were collected. They were divided into two groups by envelope lottery. Knee arthroscopy was used for the reference group of 46 patients, and they were guided to perform joint function exercises after surgery; 46 patients in the experimental group took Liuwei Gubi Pill on the basis of the reference group, and compared the efficacy of the two groups. Results: The total effective rate of treatment in the experimental group was $97.83 \%$, which was higher than the reference group's $84.78 \%$. The experimental group's VAS score at 1st month, 2nd month, and 3rd month after surgery was lower than that in the reference group. The Lysholm score of the experimental group at 1st month, 2nd month, and 3rd month after operation was significantly higher than that of the reference group, and the data of the two groups was compared by $\mathrm{P}<0.05$. Conclusion: The treatment of middle-aged and elderly patients with degenerative meniscus injury by knee arthroscopy combined with Liuwei Gubi Pill has an ideal effect, which can alleviate postoperative pain and benefit the recovery of knee function.
\end{abstract}

\section{1 introduction}

Degenerative meniscus injury has a higher incidence in middle-aged and elderly people. This is related to changes in hormone levels in the middle-aged population, which can cause knee degenerative changes and osteoporosis, and meniscus injury is more likely to occur [1]. Degenerative meniscus injury is mainly manifested by local pain and interlocking symptoms of the knee joint. It can be treated clinically by arthroscopic surgery, and usually good results can be obtained, but joint pain and slow recovery of joint function will occur, so it can add some corresponding adjuvant therapy [2]. The meniscus injury belongs to the category of TCM arthralgia. In order to ensure that patients can get better recovery after surgery, our hospital advocates the addition of Chinese medicine Liuwei Gubi Pill to promote the expression of cartilage collagen and repair of cartilage in knee joints by supplementing liver and kidney. In this study, 92 patients were treated in groups to discuss the effect and value of knee arthroscopy combined with Liuwei Gubi Pill. The report is as follows:

\section{Clinical data and methods}

A total of 92 cases of middle-aged and elderly patients with degenerative meniscus injury who were treated in our hospital from August 2018 to February 2020 were collected. According to the envelope drawing method, the research subjects were divided into two groups, 46 cases in each. The experimental group consisted of 22 male and 24 female patients, aged 45-75 years, with an average of $(58.9 \pm 6.0)$ years; body mass index was $(25.05 \pm 2.01) \mathrm{kg} / \mathrm{m} 2$. The reference group was composed of 24 male and 22 female patients, aged $44-75$ years, with an average of $(58.2 \pm 6.3)$ years; body mass index is $(24.97 \pm 2.05) \mathrm{kg} / \mathrm{m} 2$. After statistical analysis, the comparison results of the data of the two groups of patients showed $\mathrm{P}>0.05$, which is comparable to the study. The above patients all have the right of informed consent. The study has been reviewed and approved by the ethics committee.

(1) Inclusion criteria: those aged 45-75 years old, diagnosed as meniscus injury by MRI or CT examination, with joint pain and snapping performance, those with positive Maier's sign, TCM syndrome is liver and kidney deficiency with stasis syndrome.

(2) Exclusion criteria: those with meniscus injury due to trauma, those with ligament injuries or osteochondral tumors, those with rheumatic diseases, and those with a history of knee surgery are excluded.

\subsection{Clinical data}




\subsection{Method}

Reference group: The patients in this group were treated with knee arthroscopy, taken in a supine position, and combined lumbar anesthesia for the patients, and tied the tourniquet on the affected limbs. The approach is from the anterior medial and lateral approach of the knee joint, and the hyperplastic synovial tissue in the joint cavity is carefully explored and cleaned. Removal and trimming of the diseased tissue, the meniscus tissue should be retained as much as possible, and then suturing it, when suturing the medial meniscus, it should take the position of 15 degrees of knee flexion; Taking the 4th position of the knee joint when suturing the lateral meniscus, then check the joint cavity, suturing the incision, using elastic bandage to apply pressure bandage, and use the brace to fix the knee joint in the straight position. On the third day after surgery, the patient was instructed to perform functional exercises for the quadriceps and knee joints.

Experimental group: The patients in this group were combined with Liuwei Gubi Pill based on arthroscopic surgery, consisting of $40 \mathrm{~g}$ turtle shell, $30 \mathrm{~g}$ Achyranthes bidentata, 20g each of Epimedium, Bupleurum, Haitong skin, $10 \mathrm{~g}$ licorice, fried, concentrated into $50 \mathrm{~g} /$ dose water honey pill. Oral administration started at $24 \mathrm{~h}$ after surgery, $6 \mathrm{~g} /$ time, 3 times/day, for 3 consecutive months. On the third day after the operation, it should start functional exercises for the quadriceps and knee joints.

\subsection{Evaluation standard of curative effect [3]}

After 3 months, the international knee literature committee IKDC scale was used to evaluate the treatment and rehabilitation effects of the patients. When the patient's IKDC scale score is 80 points or more, it is marked effective; when the score is between 60 and 80 points, it is effective; when the score is less than 60 points, it is ineffective.

Statistical value of total effective rate of treatment $=$ (number of marked effective cases + number of effective cases) / total number of cases $\times 100 \%$.

\subsection{Evaluation index}

(1) VAS evaluation value: at the time of preoperative and at 1st month, 2nd month, and 3rd month post operation, visual analog scale (VAS) was used to evaluate the patient's knee pain, and the score range was $0--10$ points, 0 points indicate no pain symptoms, 10 points indicate severe pain [4].

(2) Lysholm score: before operation, and at 1st month, 2nd month, and 3rd month post operation, the Lysholm knee function assessment scale was used to evaluate the degree of rehabilitation of the knee function of the patient, and the score range was $0--100$ points, when the score is less than 70 points, it means that the knee function is poor [5].

\subsection{Statistical analysis}

The data obtained from the study was input into the statistical software SPSS21.0 for analysis. The manifestation of the measurement data was $( \pm \mathrm{s})$, the comparison between the groups was verified by $t$ value, and the manifestation of the count data was (n, \%). The results were verified by 2 value. When $\mathrm{P}<0.05$, there was a statistically significant difference.

\section{Research results}

\subsection{The difference between the total treatment efficiency of the two groups of patients}

The statistical value of the total treatment efficiency in the experimental group was $97.83 \%$, which was significantly higher than the reference group's $84.78 \%$. The comparison between the groups was $<0.05$; see Table 1 for details.

Table 1: Comparison of differences in total treatment efficiency between the two groups (n, \%)

\begin{tabular}{cccccc}
\hline Group & Cases & $\begin{array}{c}\text { Marked } \\
\text { Effective }\end{array}$ & Effective & Ineffective & Total efficiency \\
\hline $\begin{array}{c}\text { Experimental } \\
\text { Group }\end{array}$ & 46 & 37 & 8 & 1 & $97.83 \%(45 / 46)$ \\
Reference & 46 & 24 & 15 & 7 & $84.78 \%(39 / 46)$ \\
Group & & & & 4.9286 \\
$\chi_{\mathbf{2}}$ & & & & 0.0264 \\
\hline $\mathbf{P}$ & & & & \\
\hline
\end{tabular}

\subsection{The difference between the VAS evaluation values of the two groups of patients}

The comparison of the VAS evaluation values of the two groups of patients before treatment showed no significant

Table 2: Comparison of differences in VAS evaluation values between the two groups ( $\pm \mathrm{s}$, points)

\begin{tabular}{cccccc}
\hline Group & Cases & $\begin{array}{c}\text { Before } \\
\text { Operation }\end{array}$ & $\begin{array}{c}\text { 1st Month } \\
\text { Post-operation }\end{array}$ & $\begin{array}{c}\text { 2nd Month } \\
\text { Post-operation }\end{array}$ & $\begin{array}{c}\text { 3rd Month } \\
\text { Post-operation }\end{array}$ \\
\hline $\begin{array}{c}\text { Experimental } \\
\text { Group }\end{array}$ & 46 & $5.10 \pm 0.78$ & $3.21 \pm 0.65$ & $2.25 \pm 0.40$ & $0.74 \pm 0.18$ \\
\end{tabular}




$\begin{array}{lcc}\begin{array}{c}\text { Reference } \\ \text { Group } \\ \mathbf{t}\end{array} & 46 & 5.18 \pm 0.75 \\ \mathbf{P} & 0.5014 \\ & 0.6173 \\ \text { The difference of Lysholm score between the } \\ \text { To groups }\end{array}$

There was no significant difference in Lysholm score between the two groups of patients before treatment,

Table 3: Differences in Lysholm scores between the two groups ( \pm s, points)

\begin{tabular}{cccccc}
\hline Group & Cases & Before Operation & $\begin{array}{c}\text { 1st Month } \\
\text { Post-operation }\end{array}$ & $\begin{array}{c}\text { 2nd Month } \\
\text { Post-operation }\end{array}$ & $\begin{array}{c}\text { 3rd Month } \\
\text { Post-operation }\end{array}$ \\
\hline $\begin{array}{c}\text { Experimenta } \\
\text { I Group }\end{array}$ & 46 & $60.73 \pm 3.20$ & $71.49 \pm 3.62$ & $86.63 \pm 3.93$ & $90.21 \pm 3.80$ \\
$\begin{array}{c}\text { Reference } \\
\text { Group }\end{array}$ & 46 & $60.31 \pm 3.72$ & $65.08 \pm 3.55$ & $78.10 \pm 4.10$ & $84.48 \pm 4.02$ \\
t & & 0.5805 & 8.5746 & 10.1866 & 7.0254 \\
P & & 0.5630 & 0.0000 & 0.0000 & 0.0000 \\
\hline
\end{tabular}

\section{Discussion}

The meniscus has important physical and chemical functions in the knee joint, including bearing, absorbing shock, maintaining joint stability, protecting the articular surface, and assisting lubrication. When the knee joint is flexed at a right angle, the meniscus can carry a load of up to $85 \%$, for those with meniscus injuries, their anatomical integrity should be restored in time to promote functional rehabilitation of the knee [6].

As the middle-aged and the elderly grow older, bones will gradually degenerate and tissues will gradually wear out, which may cause the meniscus to slowly degenerate and become damaged. Arthroscopic surgery is a treatment for meniscus injury. The damaged meniscus is repaired and sutured under arthroscopy, but it is still controversial for middle-aged and elderly patients to use this therapy, because the degree of destruction of the meniscus structure in many patients is much less than that of traumatic meniscus injury, such as arthroscopy treatment, which will cause a certain degree of secondary damage. Moreover, the recovery of middle-aged and elderly patients is slow, so it is necessary to determine whether to perform arthroscopic surgery according to the degree of injury in clinical practice. If the extent of the meniscus tissue damage is very serious, arthroscopy is the first choice.

In traditional Chinese medicine, meniscus injury belongs to the category of "bone paralysis". For patients with liver and kidney deficiency syndrome, the main reason is that the liver deficiency leads to blood failure and no maintenance of the joints, resulting in joint slippage [7]; On the other hand, reduced kidney marrow causes muscle and bone dystrophy, coupled with long-term wear and tear, muscle and bone damage, which can lead to blood stasis and joint pain. To this end, our department tried to use traditional Chinese medicine for adjuvant treatment of patients. The selected drug was Liuwei Gubi Pill, and the epimedium contained therein has the effect of warming the kidney and strengthening the yang qi and the bones; Tortoise shell and Haitong skin are good medicines for liver and kidney, which have the effect of strengthening bones and muscles, and nourishing heart and blood; Gusuibu and Achyranthes bidentata can promote blood circulation and menstrual flow, and lead medicine downwards; licorice can nourish qi and harmonize various medicines; all can enhance the effect of nourishing liver and kidney, strengthening muscles and bones. Modern pharmacology confirmed [8] that the drug can reduce inflammatory factors and tumor necrosis factors in serum and joint fluid, promote cartilage repair, and relieve joint pain.

The results of this study showed that the total treatment efficiency of the experimental group was higher than that of the reference group, the postoperative VAS score was lower than that of the reference group, the Lysholm score was higher than that of the reference group, and the comparison between groups was $\mathrm{P}<0.05$, which also fully reflects the application effect and value of Liuwei Gubi Pill.

In summary, the use of knee arthroscopy combined with Liuwei Gubi Pill to treat degenerative meniscus injury in middle-aged and elderly patients has low postoperative pain and good recovery of knee function, which is worthy of promotion.

\section{Acknowledgement}

In this thesis writing, my teacher gave me a lot of guidance, so that my thesis has been comprehensively improved. Thank you for your hard work, and sincerely wish you a smooth job and good health.

\section{References:}

1. Feng Xu. "Comparison of X-ray plain film and MR imaging diagnosis of knee degenerative osteoarthropathy $[\mathrm{J}] "$ in Chinese and Foreign Medicine, 2019, 38(14): 162-164.

2. Liang Yu, Li Qiang, Guo Ai, "Comparative study of partial meniscus resection and non-surgical treatment of degenerative injury of the medial 
meniscus of the knee $[\mathrm{J}]$ " in Journal of Clinical and Experimental Medicine, 2020,19(2):205-209

3. Peng Qi, Li Xiaodong, Cao Guangjie, "Arthroscopy combined with a small needle knife to loosen the superficial layer of the medial collateral ligament of the knee to treat degenerative medial meniscus injury of the knee $[\mathrm{J}]$ " in Chinese Journal of Bone Injury, 2019, 32(12): 1090 -1093.

4. Wang Aiming, Xiao Zhiqing, Wang Fenglong, "Arthroscopy combined with exercise therapy for the treatment of non-traumatic degenerative medial meniscus injury of the knee joint $[\mathrm{J}]$ " in Chinese Journal of Medical Science, 2020,10(6):270-272,287.

5. Yan Xueliang, Chen Xiaoming, Zhang Jie, "Prognostic evaluation of arthroscopy for patients with degenerative medial meniscus injury of the knee[J]" in Chinese Journal of Endoscopy, 2018, 24(1): 39-44.
6. Chen Pengyu, Zhu Hao, Yuan Feng, "Observation on the effect of exercise therapy and arthroscopy on middle-aged degenerative meniscus injury[J]" in Chinese Journal of Bone and Joint Injury, 2019, 34(4): 401-403.

7. Wang Zheng, Liu Anping, Liang Wenwu, "Observation of the short-term curative effect of meniscus plasty combined with traditional Chinese medicine for the treatment of degenerative meniscus injury[J]" in World Science and Technology-Modernization of Traditional Chinese Medicine, 2019, 21(7): 1530- 1535.

8. Li Yuan, Hu Liekui, Peng Liping, "Clinical effect of knee arthroscopy combined with Liuwei Gubi Pill in the treatment of middle-aged and elderly patients with degenerative meniscus injury[J]" in Guangxi Medicine, 2019, 41(15): 1888-1890, 1894. 